\title{
The $\Sigma-D$ relation for Galactic planetary nebulae: Application of orthogonal fitting procedure
}

\author{
Branislav Vukotić $\dot{c}^{1}$ and Dejan Urošević $\dot{c}^{2}$ \\ ${ }^{1}$ Astronomical observatory, \\ Volgina 7, \\ 11060, Belgrade, Serbia \\ email: bvukotic@aob.rs \\ ${ }^{2}$ Dept. of Astronomy, University of Belgrade, \\ Studentski trg 16, 11000 Belgrade, Serbia \\ email: dejanu@matf.bg.ac.rs
}

\begin{abstract}
We estimate the parameters of the radio surface brightness to diameter $(\Sigma-D)$ relation for the sample of Galactic planetary nebulae (PNe). The bootstrap re-sampling and orthogonal offsets fitting procedure are applied. The orthogonal fitting procedure provides that the parameter values of $D-\Sigma$ and $\Sigma-D$ fits are invariant within the estimated uncertainties. We discuss the probability statistics of the fitted $(\log \Sigma=\log A-\beta \log D)$ relation and the resulting fit parameters which is indicative for PN distance determination.
\end{abstract}

Keywords. planetary nebulae: general, methods: numerical, methods: statistical

\section{Description}

Statistical distances to planetary nebulae $(\mathrm{PNe})$ are calculated from various types of calibrated relations. Here we calibrate radio surface brightness to diameter $(\boldsymbol{\Sigma}-\mathbf{D})$ relation. Urošević et al. (2009) derived theoretical $\Sigma-D$ in the common power law form using the interacting stellar winds model (Zhang \& Kwok 1993; Kwok 1994). $\Sigma=S / \Omega$ and $D=\theta d$, where $S$ is the source flux density, $\Omega$ is source occupied solid angle, $\theta$ is the source angular radius and $d$ is the distance to the source. Both $\Sigma$ and $D$ are calculated with non negligible uncertainties because of the uncertainties in $d, S, \theta$ and $\Omega$. Hence, $\Sigma-D$ or $D-\Sigma$ calibration should be done with orthogonal offsets instead of more commonly used vertical offsets. We applied bootstrap (resample) procedure (Efron \& Tibshirani 1993; Press et al. 2007) - require no data errors to estimate fit parameters uncertainities (similar procedure applied to supernova remnants sample in Urošević et al. 2010). In most cases the calibrating samples do not contain data errors. Additionaly, despite the extensive search we did not manage to find analytical formalism for orthogonal fit parameters uncertainties.

In Table 2 (Stanghellini et al. 2008), authors present PNe with the most reliable distances to their knowledge: some PNe have no distance error data, some have different upper and lower error and some have relative errors $>50 \%$. The compilation of $\mathrm{PNe}$ stems from the work of various authors and is not homogeneous (merely to the fact that PNe distances are determined on a case to case basis). This is indicative of many difficulties in determining reliable distances to PNe. Also it makes many problems when compiling homogeneous samples for $\Sigma-D$ calibration because data and corresponding errors are determined by different methods. This is where bootstrap comes in very handy, 
Table 1. Fit parameters values.

\begin{tabular}{|c|c|c|c|c|}
\hline $\mathrm{N} / \mathrm{A}$ & \multicolumn{2}{|c|}{$\log \Sigma=A+\beta \log D$} & $\log D=B+\alpha \log \Sigma$ \\
\hline $\mathrm{N} / \mathrm{A}$ & $A$ & $\beta$ & $B$ & $\alpha$ \\
\hline Vertical & $-20.8 \pm 0.4$ & $-2.6 \pm 0.4$ & $-6.56 \pm 0.7$ & $-0.31 \pm 0.04$ \\
Orthogonal & $-21.1 \pm 0.3$ & $-3.1 \pm 0.4$ & $-6.68 \pm 0.8$ & $-0.32 \pm 0.04$ \\
\hline
\end{tabular}
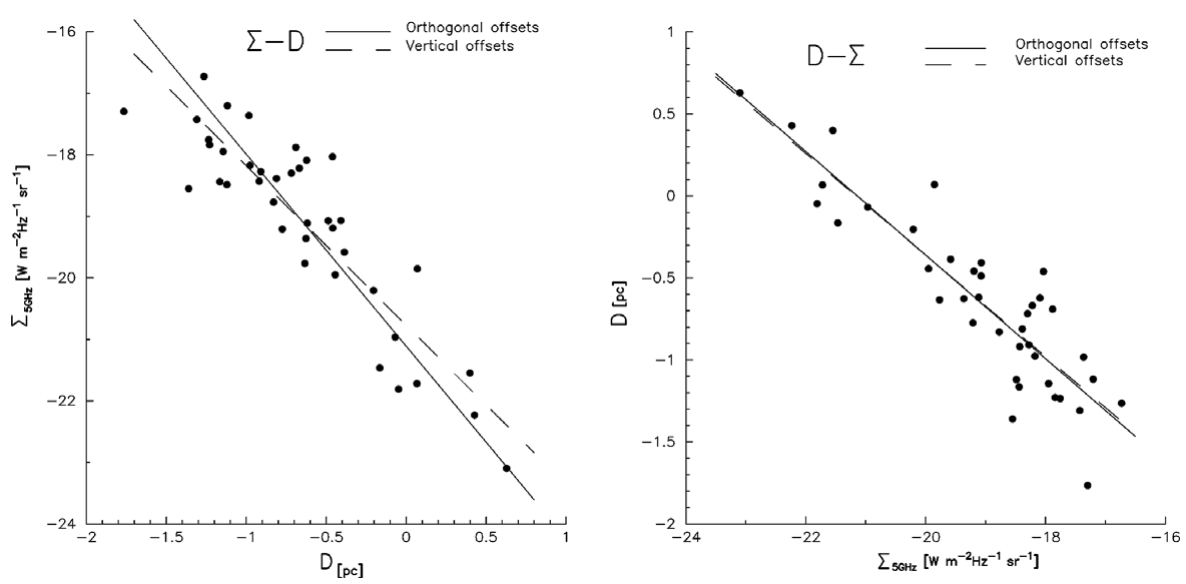

Figure 1. Calibration.

because unlike weighted fitting it does not need data errors to estimate the fit parameter uncertainties. For all Table 2 (Stanghellini et al. 2008) PNe we used radio flux density and angular radius data from Table 4 in Cahn et al. (1992). For the fitting purposes $\Sigma-D$ and $D-\Sigma$ relations were linearized as shown in Table 1 , where $A, \beta, B$ and $\alpha$ are standard linear fit parameters. It follows that $A=-B / \alpha$ and $\beta=1 / \alpha$.

Results are presented in Table 1 and Fig. 1. Flatter $\Sigma-D$ slope in the vertical offset case, compared to steeper slope of more robust orthogonal offset calibration, tends to underestimate calculated $\Sigma-D$ distances to smaller radius PNe but overestimate them for larger radius PNe. In the case of flatter slope samples vertical offsets converge to orthogonal offsets and both calibrations may be used with similar results.

\section{Acknowledgements}

Funding. This research is part of the project \#176005 "Emission Nebulae: Structure and Evolution", financed by the Ministry of Education and Science, Republic of Serbia.

\section{References}

Cahn, J. H., Kaler, J. B., \& Stanghellini, L. 1992, A\&AS, 94, 399

Efron, B. \& Tibshirani, R. 1993, An Introduction to the Bootstrap (Chapman \& Hall)

Kwok, S. 1994, PASP, 106, 344

Press, W. H., Teukolsky, S. A., Vetterling, W. T., \& Flannery, B. P. 2007, Numerical recipes in C. The art of scientific computing (Cambridge University Press, Cambridge, New York)

Stanghellini, L., Shaw, R. A., \& Villaver, E. 2008, ApJ, 689, 194

Urošević, D., Vukotić, B., Arbutina, B., Ilić, D., Filipović, M., Bojičić, I., Segan, S., \& Vidojević, S. $2009, A \mathscr{E} A, 495,537$

Urošević, D., Vukotić, B., Arbutina, B., \& Sarevska, M. 2010, ApJ, 719, 950

Zhang, C. Y. \& Kwok, S. 1993, ApJS, 88, 137 